\title{
Cheeger Sets for Rotationally Symmetric Planar Convex Bodies
}

\author{
Antonio Cañete(1)
}

\begin{abstract}
In this note we obtain some properties of the Cheeger set $C_{\Omega}$ associated to a $k$-rotationally symmetric planar convex body $\Omega$. More precisely, we prove that $C_{\Omega}$ is also $k$-rotationally symmetric and that the boundary of $C_{\Omega}$ touches all the edges of $\Omega$.
\end{abstract}

Mathematics Subject Classification. 52A10, 52A40, 28A75.

Keywords. Cheeger set, Rotationally symmetric.

\section{Introduction}

The Cheeger problem is a classical problem in Geometry widely studied in literature, with connections in many different fields. The interested reader may find in [50] some historical remarks on closely related questions (considered, among others, by J. Steiner or A. S. Besicovitch), see also [18, Problem A23]. The origin of this problem can be traced back to a paper by J. Cheeger [17], who proved in 1969 the following inequality for any bounded domain $\Omega$ in $\mathbb{R}^{n}$ (in fact, this result was stated for any compact Riemannian manifold without boundary):

$$
\lambda_{1}(\Omega) \geq \frac{h(\Omega)^{2}}{4}
$$

The author is partially supported by the MICINN project MTM2017-84851-C2-1-P, and by Junta de Andalucía grant FQM-325. 
where $\lambda_{1}(\Omega)$ is the first eigenvalue of the Laplacian on $\Omega$ (under Dirichlet conditions), and

$$
h(\Omega)=\inf _{X \subseteq \bar{\Omega}} \frac{P(X)}{V(X)},
$$

where the infimum in (2) is taken over all non-empty finite-perimeter sets $X$ contained in $\bar{\Omega}$, and $P(X)$ and $V(X)$ denote the perimeter and the volume of $X$, respectively. This constant $h(\Omega)$ is usually called the Cheeger constant of $\Omega$, and any subset $X$ contained in $\bar{\Omega}$ providing the infimum in (2) is called a Cheeger set of $\Omega$. Determining the Cheeger constant and the Cheeger sets of $\Omega$, as well as their general properties, is the main core of the classical Cheeger problem.

This question, intimately related to the classical isoperimetric problem (see, for instance, the introductory texts [40,45]), has applications in very numerous distinct settings. As a sample, we briefly enumerate some of them. It is well known that the Cheeger constant is the limit of the sequence of first eigenvalues of the $p$-Laplacian (with Dirichlet conditions) of a bounded domain when $p$ tends to 1 , see [26]. This result was later extended to the sequence of second eigenvalues in [41] by using the notion of higher Cheeger constants, see [7]. In the field of image regularization, an approach for removing noise in a given image [46] is strongly connected to the two-dimensional Cheeger problem, as described in [32, §. 2.3], see also [3]. Additionally, the Cheeger constant explicitly appears in the condition on the pressure needed for breaking down a planar plate [29], and also in some maximal flow-minimal cuts planar problems [51] (with further applications in the field of medical imaging). Moreover, for the problem of finding hypersurfaces in $\mathbb{R}^{n}$ with prescribed (constant) mean curvature [22], the Cheeger constant is involved and provides some equilibrium capillary free-hypersurfaces in certain cases (see $[32, \S .2 .2]$ ). The interested reader may find more details of these (and some others) applications in [42, $\S .7]$ or $[32, \S .2]$. We can even find analogues to the Cheeger problem in graph theory [53].

For a given bounded domain $\Omega$ in $\mathbb{R}^{n}$, finding the Cheeger constant and the Cheeger sets is, in general, a hard task. However, questions such as the existence and uniqueness of the Cheeger sets, as well as intrinsic properties of these sets, have been well studied in different works. Our Lemma 1 collects some of the main results in this direction, but we mention here that the existence of Cheeger sets is assured in our setting due to the boundedness of $\Omega$, and therefore the infimum in (2) is always attained under this hypothesis. Moreover, if $\Omega$ is convex, we have uniqueness of the associated Cheeger set, which will be also convex with smooth boundary.

In the two-dimensional case, the Cheeger problem is more treatable, although the complete determination of the Cheeger sets is only known for some specific situations. Among all the papers in this context, we remark a particular one by B. Kawohl and T. Lachand-Robert [27], where we can find some 
interesting characterizations of the Cheeger sets for planar convex sets. One of these results establishes a condition on a planar convex set $\Omega$ for assuring that the Cheeger set of $\Omega$ is $\Omega$ itself (in other words, a condition on $\Omega$ to be calibrable, see [4]), in terms of an inequality involving the curvature of the boundary of $\Omega$ [27, Th. 2] (see also [47, Crit. 1.5], and [4, Co. 1]). This result can be applied to the circle, as well as the stadium domain and certain ellipses. On the other hand, [27, Th. 1] gives a useful description of the Cheeger set of any planar convex set (specifying also the value of the Cheeger constant) as a Minkowski sum in terms of the inner parallel set (see also [6], [52, Th. 3.32]). We will use this result, which is stated in Theorem 1, in our Sect. 3.

The Cheeger problem in the class of convex polygons is another question of particular interest, treated for instance in [27, §. 4 and $\S .5]$ and in [10]. For a given convex polygon $P$, standard properties yield that its associated Cheeger set $C_{P}$ will be bounded by certain line segments and certain circular arcs, see Lemma 1. Taking into account this, it may happen that the boundary $\partial C_{P}$ of $C_{P}$ touches all the sides of the polygon $P$. In that case, $P$ is called Cheeger-regular polygon. Some examples of this situation are triangles and rectangles, and a necessary and sufficient (analytic and geometrical) condition is established in [27, Th. 3]. Otherwise, if $\partial C_{P}$ does not touch all the sides of $P$, then $P$ is a Cheeger-irregular polygon (as commented in [27], a quadrilateral with a very small side is Cheeger-irregular). Some remarkable consequences of this are the following: for a Cheeger-regular convex polygon $P$, its associated Cheeger set can be obtained by rounding all the corners of $P$, and for any general convex polygon, a direct algorithm for determining its Cheeger set is described in $[27, \S .5]$.

In this note, we will focus on the class of $k$-rotationally symmetric planar convex bodies. This class of sets has been considered in the last years for some partitioning problems involving the maximum relative diameter functional (see [12] and references therein). In this setting, we will introduce the notion of $k$ Cheeger-regular set, which is inspired by the notion of Cheeger-regular polygon treated in $[27, \S .4]$. We will see that we can properly define the concepts of dots and edges for any $k$-rotationally symmetric planar convex body $\Omega$, which will play the role of the vertices and the sides of a polygon. We will prove in Theorem 2 that any set in this class is $k$-Cheeger-regular (that is, the boundary of the corresponding Cheeger set $C_{\Omega}$ touches all the edges of $\Omega$ ), and additionally, we will see in Corollary 1 that the $k$-rotational symmetry of $\Omega$ is inherited by $C_{\Omega}$. We finish this note with Sect. 4 , where we list some variants and open questions related to the Cheeger problem.

Notation. Throughout this paper, we will denote by $d$ the Euclidean distance in the plane. For a given planar bounded set $X, P(X)$ will stand for the perimeter of $X$ (that is, the one-dimensional Hausdorff measure of $\partial X$ whenever $X$ is convex), and $A(X)$ will stand for the Euclidean area of $X$. Moreover, the addition of two planar sets must be understood as the classical 
Minkowski addition. We also recall that a planar body is, as usual, a planar compact set.

\section{Preliminaries}

\subsection{Some Generalities on the Cheeger Problem}

As stated in the Introduction, the Cheeger problem has been deeply studied in the literature. We collect in Lemma 1 some well-known results, which can be found in many different texts (see for instance $[2,32,42]$ and references therein).

Lemma 1. Let $\Omega$ be a planar convex body. Then, there exists a Cheeger set $C_{\Omega}$ of $\Omega$. Moreover,

i) $C_{\Omega}$ is unique, convex and of class $C^{1,1}$.

ii) $C_{\Omega}$ touches the boundary of $\Omega$.

iii) $C_{\Omega}$ is an isoperimetric region in $\Omega$ for the area it encloses.

iv) The pieces of the boundary of $C_{\Omega}$ in the interior of $\Omega$ are circular arcs of curvature $1 / h(\Omega)$, where $h(\Omega)$ is the Cheeger constant of $\Omega$.

We can find an interesting characterization of the Cheeger set for any planar convex body $\Omega$ in [27]. For any $t>0$, denote by $\Omega^{t}$ the inner parallel set of $\Omega$ at distance $t$, that is,

$$
\Omega^{t}=\{x \in \Omega: \operatorname{dist}(x, \partial \Omega)>t\} .
$$

Then we have the following result.

Theorem 1. ([27, Th. 1]) Let $\Omega$ be a planar convex body. Then, there exists a unique value $s>0$ such that $A\left(\Omega^{s}\right)=\pi s^{2}$. We also have that $h(\Omega)=1 / s$ and the Cheeger set of $\Omega$ is $C_{\Omega}=\Omega^{s}+s B_{1}$, where $B_{1}$ is the Euclidean unit disk.

Theorem 1 gives a nice geometrical characterization of the Cheeger constant in this convex setting, by means of the inner parallel set $\Omega^{s}$ of $\Omega$ which encloses the same area as the planar disk of radius $s$, and shows that $C_{\Omega}$ is precisely the Minkowski addition of that inner parallel set and that disk. From this result we also deduce that the part of $\partial C_{\Omega}$ contained in the interior of $\Omega$ is a union of circular arcs of radius $s$, and hence $h(\Omega)$ (which coincides with $1 / s$ ) can be identified as the curvature of $\partial C_{\Omega}$ in the interior of $\Omega$ (see $[52$, Th. 3.32]).

\subsection{The Class of $\boldsymbol{k}$-Rotationally Symmetric Planar Convex Bodies}

Given $k \in \mathbb{N}, k \geq 2$, a planar convex body $\Omega$ is said to be $k$-rotationally symmetric if there exists a point $p \in \Omega$ such that $\Omega$ is invariant under the rotation $\theta_{k}$ of angle $2 \pi / k$ centered at $p$ (the point $p$ is usually called the center of symmetry of $\Omega)$. Note that, in that case, $\theta_{k}(\partial \Omega)=\partial \Omega$. Some examples of this type of sets are the regular polygons, or the regular Reuleaux polygons with an odd number of vertices, see Figure 1. 

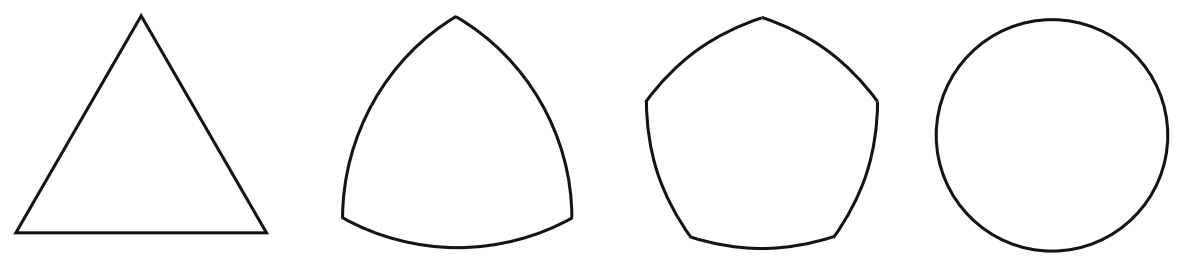

FIGURE 1. Some examples of $k$-rotationally symmetric planar convex bodies: an equilateral triangle and a regular Reuleaux triangle $(k=3)$, a regular Reuleaux pentagon $(k=5)$, and a circle
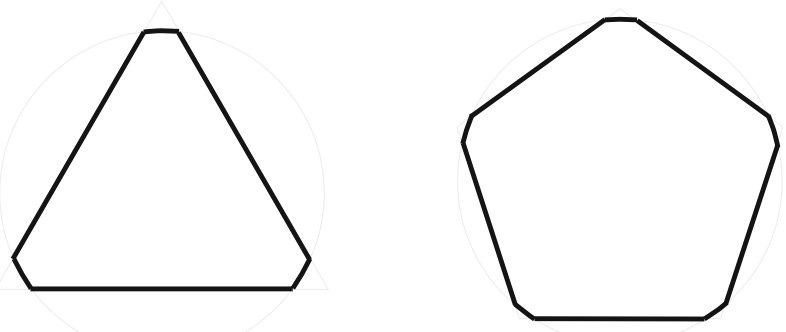

Figure 2 . Two $k$-rotationally symmetric planar convex bodies, for $k=3$ and $k=5$

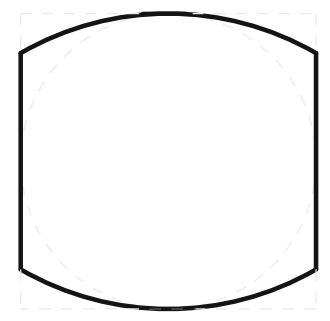

FiguRE 3. A 2-rotationally symmetric planar convex body

Figure 2 shows two interesting $k$-rotationally symmetric planar convex bodies, for $k=3$ and $k=5$. They are obtained by interesecting the unit disk with a certain equilateral triangle, and with a certain regular pentagon. These sets appear as the optimal bodies for an optimization division problem involving the maximum relative diameter functional [11, Th. 5.1]. Additionally, the set from Figure 3 is another remarkable example of this type of sets for $k=2$. It provides an example where the standard bisection is not minimizing for the maximum relative diameter functional, see details in [12, Ex. 3]. 

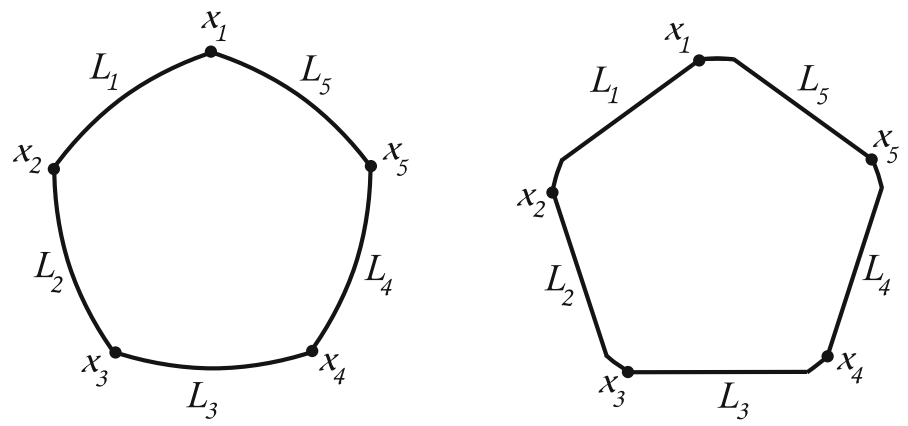

Figure 4. Dots and edges of two different 5-rotationally symmetric planar convex bodies

We will now define the notions of dots and edges of a $k$-rotationally symmetric planar convex body $\Omega$ (keeping certain analogy with the vertices and the sides of polygons). Let $R>0$ be the circumradius of $\Omega$ (that is, the radius of the smallest ball containing $\Omega$ ), and let $p$ be the center of symmetry of $\Omega$. Then, there exist $x_{1}, \ldots, x_{k} \in \partial \Omega$ such that $d\left(x_{i}, p\right)=R$, which can be further chosen to be $k$-rotationally symmetric (observe that the circumradius is necessarily attained by a point $x_{1} \in \partial \Omega$, and by applying repeatedly the rotation $\theta_{k}$ we will obtain the rest of the points. More precisely, $x_{i}=\theta_{k}^{i-1}\left(x_{1}\right)$, for $i=2, \ldots, k)$. We will call dots of $\Omega$ any choice of these points (notice that the set of dots may not be unique for $\Omega$ ). Moreover, for a given set of $\operatorname{dots} x_{1}, \ldots, x_{k}$ of $\Omega$, an edge of $\Omega$ will be any piece of $\partial \Omega$ delimited by two consecutive dots. This implies that $\Omega$ will have $k$ edges, namely $L_{i}=\left[x_{i}, x_{i+1}\right]$, $i=1, \ldots, k$, with the convention that $x_{k+1}=x_{1}$, see Figure 4 . Note that the edges $L_{1}, \ldots, L_{k}$ of $\Omega$ are placed along $\partial \Omega$ in the counterclock-wise order.

Remark 1 . Let $P$ be a $k$-rotationally symmetric convex polygon with $n$ sides (and $n$ vertices). In this case, we want to stress that the notions of sides and edges will not coincide, in general, as well as the notions of vertices and dots. For instance, if $P$ is a regular polygon, then it will be clearly $n$-rotationally symmetric, and its sides and edges will be the same. However, if we consider an equilateral triangle and we (slightly) cut its vertices symmetrically (see Figure 5), we will obtain a non-regular hexagon which is 3-rotationally symmetric, that is, with six sides and three edges. In this setting, it is not difficult to check that $k \leq n$ : the circumradius of $P$ is always given by the distance between its center of symmetry and a vertex of $P$, which implies that any dot of $P$ is indeed a vertex. And consequently, taking into account the previous definition of an edge, it follows that any edge of $P$ will be composed by one or several sides of $P$ (more precisely, each edge of $P$ will contain $n / k$ sides).

We can now define the notion of $k$-Cheeger-regular set in the class of $k$-rotationally symmetric planar convex bodies. 


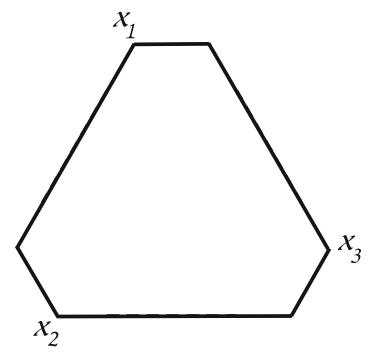

FigurE 5. A 3-rotationally symmetric hexagon

Definition 1. Let $\Omega$ be a $k$-rotationally symmetric planar convex body, with dots $x_{1}, \ldots, x_{k}$ and edges $L_{1}, \ldots, L_{k}$. Let $C_{\Omega}$ be the Cheeger set of $\Omega$. Then, $\Omega$ is called $k$-Cheeger-regular if the boundary of $C_{\Omega}$ touches all the edges of $\Omega$.

Remark 2. Definition 1 does not depend on the choice of the dots of $\Omega$.

Remark 3. Consider a $k$-rotationally symmetric convex polygon $P$. We anticipate that $P$ is $k$-Cheeger-regular, by virtue of Theorem 2 below. Let us assume that $P$ is Cheeger-regular, following the definition given in $[27, \S$. 4]. Then, the boundary of its associated Cheeger set $C_{P}$ will touch all the sides of $P$. Taking into account Remark 1, it follows that $\partial C_{P}$ will also touch all the edges of $P$, and therefore $P$ will be $k$-Cheeger-regular. On the other hand, we can find examples of $k$-Cheeger-regular convex polygons which are not Cheeger-regular. For instance, consider a very narrow rhombus where we have cut slightly two vertices symmetrically, see Figure 6 . This convex polygon is 2-rotationally symmetric, and so 2-Cheeger-regular (by Theorem 2), but it can be checked that it is not Cheeger-regular (the condition from [27, Th. 3] is not satisfied if the set is narrow enough and the cutting is very small). This means that the notion of $k$-Cheeger-regular, when restricted to the family of $k$-rotationally symmetric convex polygons, is weaker than the Cheeger-regular notion introduced in $[27$, $\S .4]$.

\section{Main Results}

In this section we prove our main results, by using Theorem 1 . Theorem 2 shows that any $k$-rotationally symmetric planar convex body $\Omega$ is $k$-Cheeger-regular, and Corollary 1 assures that the Cheeger set of $\Omega$ is always $k$-rotationally symmetric (that is, in this setting, the rotational symmetry is inherited by the Cheeger set).

Theorem 2. Let $\Omega$ be a $k$-rotationally symmetric planar convex body. Then, $\Omega$ is $k$-Cheeger-regular, that is, the boundary of its Cheeger set $C_{\Omega}$ touches all the edges of $\Omega$. 

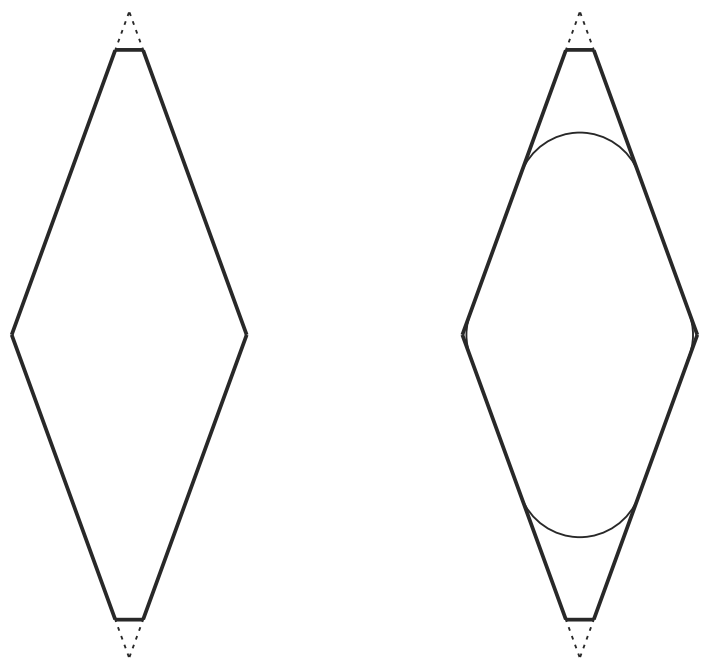

FiguRE 6. A 2-rotationally symmetric convex polygon which is 2-Cheeger regular, but not Cheeger regular. The associated Cheeger set is depicted in the right-hand side

Proof. Fix $q \in \partial C_{\Omega} \cap \partial \Omega$ (recall that $\partial C_{\Omega}$ touches necessarily $\partial \Omega$ ). We can assume that $q$ lies in the edge $L_{1}$ of $\Omega$. Taking into account the characterization of $C_{\Omega}$ from Theorem 1 , it follows there exists $s>0$ such that $q=z+s v$, for certain $z \in \partial \Omega^{s}$ and $v \in \mathbb{S}^{1}$, and by applying the rotation $\theta_{k}$, we will obtain that $\theta_{k}(q)=\theta_{k}(z)+s \theta_{k}(v)$. Observe that $\theta_{k}(q) \in L_{2}$ and $\theta_{k}(v) \in \mathbb{S}^{1}$. Let us prove that $\theta_{k}(z) \in \partial \Omega^{s}$.

Since $z \in \partial \Omega^{s}$, we have that $\operatorname{dist}(z, \partial \Omega)=\min \{d(z, w): w \in \partial \Omega\}=s$. Clearly, $d(z, w)=d\left(\theta_{k}(z), \theta_{k}(w)\right)$ for any $w \in \partial \Omega$. Since $\theta_{k}$ is bijective when restricted to $\partial \Omega$, it follows that

$$
s=\min \{d(z, w): w \in \partial \Omega\}=\min \left\{d\left(\theta_{k}(z), w\right): w \in \partial \Omega\right\},
$$

which implies that $\theta_{k}(z) \in \partial \Omega^{s}$. Therefore $\theta_{k}(q)=\theta_{k}(z)+s \theta_{k}(v) \in \partial \Omega^{s}+$ $s B_{1}=\partial C_{\Omega}$, using again Theorem 1. This gives that $\partial C_{\Omega} \cap L_{2} \neq \emptyset$, and repeating the argument, it yields that $\partial C_{\Omega} \cap L_{i} \neq \emptyset$, for $i=1, \ldots, k$, which means that $\partial C_{\Omega}$ touches all the edges of $\Omega$, as stated.

Corollary 1. Let $\Omega$ be a k-rotationally symmetric planar convex body. Then, its Cheeger set $C_{\Omega}$ is k-rotationally symmetric.

Proof. It suffices to prove that $\theta_{k}\left(C_{\Omega}\right) \subset C_{\Omega}$. Let $q$ be an arbitrary point in $C_{\Omega}$. In view of Theorem 1 , for $s=1 / h(\Omega)$, we will have that $q=z+s v$, for certain $z \in \Omega^{s}$ and $v \in B_{1}$. Then $\theta_{k}(q)=\theta_{k}(z)+s \theta_{k}(v)$. It is clear that $\theta_{k}(v) \in B_{1}$, and applying an analogous reasoning to the one from the proof of Theorem 2, we have that $\theta_{k}(z) \in \Omega^{s}$. Thus $\theta_{k}(q) \in \Omega^{s}+s B_{1}=C_{\Omega}$, which concludes the proof. 
Remark 4. An alternative proof of Corollary 1 is the following. It is clear that $\theta_{k}\left(C_{\Omega}\right) \subseteq \theta_{k}(\Omega)=\Omega$, and also that $P\left(\theta_{k}\left(C_{\Omega}\right)\right)=P\left(C_{\Omega}\right)$ and $A\left(\theta_{k}\left(C_{\Omega}\right)\right)=$ $A\left(C_{\Omega}\right)$. This implies that $\theta_{k}\left(C_{\Omega}\right)$ is also a Cheeger set of $\Omega$. Taking into account that the Cheeger set of $\Omega$ is unique by Lemma 1 , we conclude that $\theta_{k}\left(C_{\Omega}\right)$ coincides with $C_{\Omega}$, which gives that $C_{\Omega}$ is $k$-rotationally symmetric.

Remark 5. The reader may compare our Corollary 1 with [8, Lemma 2.3], where it is proved that for a convex bounded domain $\Omega$ in $\mathbb{R}^{n}, n \geq 3$, which is also rotationally invariant (in the sense that $\Omega$ is invariant under the rotation about a fixed axis), the corresponding Cheeger set inherits the same rotational invariance.

Remark 6. The key points in the proofs of Theorem 2 and Corollary 1 are the characterization of the Cheeger sets from Theorem 1 and the fact that $\theta_{k}$ is bijective. We note that an analogous characterization has been obtained for a wider family of sets, not necessarily convex ( [33, Th. 1.4], see also [35]): if $\Omega$ is a Jordan domain (with $A(\partial \Omega)=0$ ) without necks of radius $1 / h(\Omega)$, then its associated maximal Cheeger set can be expressed as in Theorem 1 (using $\overline{\Omega^{s}}$ instead of $\Omega^{s}$ ). If we further assume that $\Omega$ is $k$-rotationally symmetric (not necessarily convex) and star-shaped with respect to its center of symmetry, it follows that $\theta_{k}$ is bijective and the proofs of Theorem 2 and Corollary 1 will work in the same way for the corresponding maximal Cheeger set of $\Omega$ (recall that the lack of convexity implies that the Cheeger set is not unique, in general). In this setting, [34, Ex. 4.7] shows an interesting 2-rotationally symmetric planar (non-convex) star-shaped body with an entire two-parameter family of associated Cheeger sets, not all of them being 2-rotationally symmetric.

\section{Further Comments}

We finish with some general comments of interest on the Cheeger problem.

Remark 7. An interesting variant of the Cheeger problem, stated in [18, Problem A23], consists of minimizing the quotient $P(X)^{\alpha} / A(X)$, for $\alpha>0$, over all subsets $X$ contained in a given planar (convex) body $\Omega$, looking also for the optimal sets. The reciprocal problem of minimizing $P(X) / V(X)^{\alpha}$ (over subsets $X$ of a given domain $\Omega$ in $\mathbb{R}^{n}$ ) has been considered in [19], where a sharp lower bound for the corresponding Cheeger constant is given (see also [5] for some other lower and upper bounds), and in [44], where the general properties of this problem are obtained, and the cases of rectangles and (2-dimensional) curved strips are studied in detail.

Remark 8. The nonlocal/fractional Cheeger problem is another variant dealt with in literature, where the classical fractional perimeter is considered. This question has been treated in [9], see also [38, Ch. 5]. 
Remark 9. We also have, as a sort of extension of the problems described in Remark 7, the so-called generalized Cheeger problem, where two different positive functions on a planar bounded domain are used for weighting the perimeter and area functionals. This is partially studied in [24], with interesting applications in the framework of landslides modeling, since the generalized Cheeger constant coincides with a certain coefficient related to the stability of landslides (see [24] and references therein). In that work, among other things, the Cheeger sets and Cheeger constants for this generalized problem are described when considering planar rectangles and a particular family of weights which depend only on one variable $[24$, Th. 7 and Re. 5.E]. These results may suggest that the study of this generalized Cheeger problem in different domains and with different weights (also known as densities, which have been deeply considered in the last years, see [39]) is an interesting question which could reveal applications in other settings (for instance, there is a strong connection with the existence of rotationally invariant Cheeger sets in domains of $\mathbb{R}^{n}$, see [8, Re. 5.1]). We further point out that the generalized Cheeger problem in $\mathbb{R}^{n}$ with the Gaussian density weighting the perimeter and volume functionals has been considered in [16] and in [25] (see also [1,37,48], where some existence, regularity and other interesting results on the generalized Cheeger sets for different densities have been obtained).

Remark 10. In the anisotropic case (that is, when the perimeter is given by means of a general non-Euclidean norm), a characterization of the Cheeger set associated to a planar convex bounded domain can be found in [28, Th. 5.1], following the same spirit of [27, Th. 1]. Taking this into account, an interesting direction for future work could be studying whether our results hold in this more general situation. In this setting, we think that there will be a strong dependence on the structure of the corresponding Wulff shape. For instance, in order to extend our Corollary 1 when considering a $k$-rotationally symmetric convex polygon $\Omega$, it seems necessary that the Wulff shape $W$ is a $\widetilde{k}$-rotationally symmetric convex polygon, satisfying certain divisibility relation between $k$ and $\widetilde{k}$ (and moreover, different behaviors may appear depending on the fact that $\Omega$ and $W$ have some parallel sides).

Remark 11. An additional related question is posing the Cheeger problem for the other classical geometric magnitudes, that is, minimizing the quotient $F(X) / A(X)$ over all subsets $X$ contained in a given planar convex body $\Omega$, where $F(X)$ stands for the minimal width, or the circumradius, or the inradius of $X$. This was also suggested in [18, Problem A23], and we have not found any related reference in literature. This is surprising for us, because the relations between pairs (and triplets) of these classical magnitudes have been largely studied for a long time [49]. Incidentally, we note that the BlaschkeSantaló diagram for the triplet $(P(\cdot), A(\cdot), h(\cdot))$ for certain families of planar sets has been recently determined [20], as well as the one for the triplet 
$(r(\cdot), A(\cdot), h(\cdot))$ for the family of planar convex bodies [21], where $r$ denotes the classical inradius functional.

Remark 12. Among all the polygons of (at most) $n$ sides enclosing a prescribed quantity of area, it is known that the regular one provides the minimum possible value for the Cheeger constant [10]. This result, whose stability complement can be found in [14], is strongly related to a conjecture by Pólya and Szegó on the polygon (of $n$ sides and prescribed enclosed area) minimizing the first eigenvalue of the Laplacian [43]. On the other hand, it has been proved that the Reuleaux triangle maximizes the Cheeger constant among all planar convex bodies with (prescribed) constant width [23, Th. 1.1] (also including the computation of that maximum value, see $[23, \S .2 .2])$.

Remark 13. We want to stress that the Cheeger problem has been also treated in the non-convex planar setting, as commented in Remark 6. Some references which cover quite well this case are [31,33-36,47]. In higher dimensions, there are not so many references, and the reader may check $[13,15,30]$. Recall that the uniqueness of the Cheeger set is not assured in this non-convex setting, as shown in [27] by describing a particular example.

Remark 14. As previously commented, the Cheeger problem has been well studied during the last decade in some families of sets with certain characteristics (for instance, planar curved strips [31,34], or rotationally symmetric domains [8]). This reveals the importance of determining appropriately the setting for studying this problem.

\section{Acknowledgements}

The author would like to thank G. P. Leonardi for his help during the elaboration of this note, and the anonymous reviewers, whose comments and suggestions have undoubtedly improved the final version.

Funding Open Access funding provided thanks to the CRUE-CSIC agreement with Springer Nature.

\section{Declarations}

Conflict of interest The author declares that he has no conflict of interest.

Open Access. This article is licensed under a Creative Commons Attribution 4.0 International License, which permits use, sharing, adaptation, distribution and reproduction in any medium or format, as long as you give appropriate credit to the original author(s) and the source, provide a link to the Creative Commons licence, and indicate if changes were made. The images or other third party material in this article are included in the article's Creative Commons licence, unless indicated otherwise in a credit line to the material. If material is not included in the article's 
Creative Commons licence and your intended use is not permitted by statutory regulation or exceeds the permitted use, you will need to obtain permission directly from the copyright holder. To view a copy of this licence, visit http://creativecommons. org/licenses/by/4.0/.

\section{References}

[1] Alvino, A., Brock, F., Chiacchio, F., Mercaldo, A., Posteraro, M.R.: Some isoperimetric inequalities with respect to monomial weights. ESAIM Control Optim. Calc. Var. 27, 29 (2021). (Paper No. S3)

[2] Alter, F., Caselles, V.: Uniqueness of the Cheeger set of a convex body. Nonlinear Anal. 70(1), 32-44 (2009)

[3] Alter, F., Caselles, V., Chambolle, A.: Evolution of characteristic functions of convex sets in the plane by the minimizing total variation flow. Interfaces Free Bound. 7(1), 29-53 (2005)

[4] Alter, F., Caselles, V., Chambolle, A.: A characterization of convex calibrable sets in $\mathbb{R}^{N}$. Math. Ann. 332(2), 329-366 (2005)

[5] Avinyo, A.: Isoperimetric constants and some lower bounds for the eigenvalues of the $p$-Laplacian. Nonlinear Anal. 30(1), 177-180 (1997)

[6] Besicovitch, A.S.: Variants of a classical isoperimetric problem. Quart. J. Math. Oxford Ser. (2) 3, 42-49 (1952)

[7] Bobkov, V., Parini, E.: On the higher Cheeger problem. J. Lond. Math. Soc. (2) 97(3), 575-600 (2018)

[8] Bobkov, V., Parini, E.: On the Cheeger problem for rotationally invariant domains, ahead of print in Manuscripta Math. (2020)

[9] Brasco, L., Lindgren, E., Parini, E.: The fractional Cheeger problem. Interfaces Free Bound. 16(3), 419-458 (2014)

[10] Bucur, D., Fragalà, I.: A Faber-Krahn inequality for the Cheeger constant of $N$-gons. J. Geom. Anal. 26(1), 88-117 (2016)

[11] Cañete, A., Schnell, U., Segura Gomis, S.: Subdivisions of rotationally symmetric planar convex bodies minimizing the maximum relative diameter. J. Math. Anal. Appl. 435(1), 718-734 (2016)

[12] Cañete, A., Segura Gomis, S.: Bisections of centrally symmetric planar convex bodies minimizing the maximum relative diameter. Mediterr. J. Math. 16(6), 19 (2019). (Paper No. 151)

[13] Caroccia, M., Ciani, S.: Dimensional lower bounds for contact surfaces of Cheeger sets. J. Math. Pures Appl. (2021)

[14] Caroccia, M., Neumayer, R.: A note on the stability of the Cheeger constant of $N$-gons. J. Convex Anal. 22(4), 1207-1213 (2015)

[15] Caselles, V., Chambolle, A., Novaga, M.: Some remarks on uniqueness and regularity of Cheeger sets. Rend. Semin. Mat. Univ. Padova 123, 191-201 (2010)

[16] Caselles, V., Miranda, M., Jr., Novaga, M.: Total variation and Cheeger sets in Gauss space. J. Funct. Anal. 259(6), 1491-1516 (2010) 
[17] Cheeger, J.: A lower bound for the smallest eigenvalue of the Laplacian, Problems in Analysis: A Symposium in Honor of Salomon Bochner, 195-199. Princeton Univ. Press, Princeton, New Jersey (1970)

[18] Croft, H.T., Falconer, K.J., Guy, R.K.: Unsolved Problems in Geometry. Springer, New York (1991)

[19] Figalli, A., Maggi, F., Pratelli, A.: A note on Cheeger sets. Proc. Am. Math. Soc. 137(6), 2057-2062 (2009)

[20] Ftouhi, I.: Complete systems of inequalities relating the perimeter, the area and the Cheeger constant of planar domains, preprint (2021). https://hal. archives-ouvertes.fr/hal-03006019

[21] Ftouhi, I.: On the Cheeger inequality for convex sets. J. Math. Anal. Appl. 504(2), 26 (2021). (Paper No. 125443)

[22] Giusti, E.: On the equation of surfaces of prescribed mean curvature. Existence and uniqueness without boundary conditions. Invent. Math. 46(2), 111137 (1978)

[23] Henrot, A., Lucardesi, I.: A Blaschke-Lebesgue theorem for the Cheeger constant, preprint (2020). arxiv:2011.07244

[24] Ionescu, I.R., Lachand-Robert, T.: Generalized Cheeger sets related to landslides. Calc. Var. Part. Differ. Equ. 23(2), 227-249 (2005)

[25] Julin, V., Saracco, G.: Quantitative lower bounds to the Euclidean and Gaussian Cheeger constants. Ann. Fenn. Math. 46(2), 1071-1087 (2021)

[26] Kawohl, B., Fridman, V.: Isoperimetric estimates for the first eigenvalue of the p-Laplace operator and the Cheeger constant. Comment. Math. Univ. Carolin. 44, 659-667 (2003)

[27] Kawohl, B., Lachand-Robert, T.: Characterization of Cheeger sets for convex subsets of the plane. Pacific J. Math. 225(1), 103-118 (2006)

[28] Kawohl, B., Novaga, M.: The $p$-Laplace eigenvalue problem as $p \rightarrow 1$ and Cheeger sets in a Finsler metric. J. Convex Anal. 15(3), 623-634 (2008)

[29] Keller, J.B.: Plate failure under pressure. SIAM Rev. 22(2), 227-228 (1980)

[30] Krejčiř́́k, D., Leonardi, G.P., Vlachopulos, P.: The Cheeger constant of curved tubes. Arch. Math. (Basel) 112(4), 429-436 (2019)

[31] Krejčiř́ik, D., Pratelli, A.: The Cheeger constant of curved strips. Pacific J. Math. 254(2), 309-333 (2011)

[32] Leonardi, G.P.: An overview on the Cheeger problem, New trends in Shape Optimization. In: International Series of Numerical Mathods vol. 166, 117-139. Birkhäuser/Springer, Cham (2015)

[33] Leonardi, G.P., Neumayer, R., Saracco, G.: The Cheeger constant of a Jordan domain without necks. Calc. Var. Part. Differ. Equ. 56(6), 29 (2017). (Paper No. 164)

[34] Leonardi, G.P., Pratelli, A.: On the Cheeger sets in strips and non-convex domains. Calc. Var. Part. Differ. Equ. 55(1), 28 (2016). (Art. 15)

[35] Leonardi, G.P., Saracco, G.: Minimizers of the prescribed curvature functional in a Jordan domain with no necks. ESAIM Control Optim. Calc. Var. 26, 20 (2020). (Paper No. 76) 
[36] Leonardi, G.P., Saracco, G.: Two examples of minimal Cheeger sets in the plane. Ann. Mat. Pura Appl. 197(5), 1511-1531 (2018)

[37] Li, Q., Torres, M.: Morrey spaces and generalized Cheeger sets. Adv. Calc. Var. 12(2), 111-133 (2019)

[38] Mazón, J.M., Rossi, J.D., Toledo, J.J.: Nonlocal Perimeter. Curvature and Minimal Surfaces for Measurable Sets. Frontiers in Mathematics. Birkhäuser/Springer, Cham (2019)

[39] Morgan, F.: Manifolds with density. Notices Am. Math. Soc. 52(8), 853-858 (2005)

[40] Osserman, R.: The isoperimetric inequality. Bull. Am. Math. Soc. 84(6), 11821238 (1978)

[41] Parini, E.: The second eigenvalue of the $p$-Laplacian as $p$ goes to 1, Int. J. Differ. Equ. (2010), Art. ID 984671, 23

[42] Parini, E.: An introduction to the Cheeger problem. Surv. Math. Appl. 6, 9-21 (2011)

[43] Pólya, G., Szegő, G.: Isoperimetric Inequalities in Mathematical Physics. Annals of Mathematics Studies, no. 27, Princeton Univ. Press, Princeton, New Jersey (1951)

[44] Pratelli, A., Saracco, G.: On the generalized Cheeger problem and an application to 2d strips. Rev. Mat. Iberoam. 33(1), 219-237 (2017)

[45] Ritoré, M., Ros, A.: Some updates on isoperimetric problems. Math. Intelligencer 24(3), 9-14 (2002)

[46] Rudin, L.I., Osher, S., Fatemi, E.: Nonlinear total variation based noise removal algorithms. Phys. D 60(1-4), 259-268 (1992)

[47] Saracco, G.: A sufficient criterion to determine planar self-Cheeger sets. J. Convex Anal. 28, 3 (2021)

[48] Saracco, G.: Weighted Cheeger sets are domains of isoperimetry. Manuscripta Math. 156(3-4), 371-381 (2018)

[49] Scott, P.R., Awyong, P.W.: Inequalities for convex sets. JIPAM J. Inequal. Pure Appl. Math. 1(1), 6 (2000). (Art. 6)

[50] Singmaster, D., Souppouris, D.J.: A constrained isoperimetric problem. Math. Proc. Camb. Philos. Soc. 83(1), 73-82 (1978)

[51] Strang, G.: Maximal flow through a domain. Math. Program. 26(2), 123-143 (1983)

[52] Stredulinsky, E., Ziemer, W.P.: Area minimizing sets subject to a volume constraint in a convex set. J. Geom. Anal. 7(4), 653-677 (1997)

[53] Tan, J.: On Cheeger inequalities of a graph. Discrete Math. 269(1-3), 315-323 (2003)

Antonio Cañete

Universidad de Sevilla

Sevilla

Spain

e-mail: antonioc@us.es 
Received: February 18, 2021.

Accepted: October 6, 2021.

Publisher's Note Springer Nature remains neutral with regard to jurisdictional claims in published maps and institutional affiliations. 